\title{
Astrometric Speckle Interferometry for the Amateur
}

\author{
N. H. TURNER, D. J. BARRY, \& H. A. McALISTER \\ Center for High Angular Resolution Astronomy, Georgia State University, \\ Atlanta, Georgia 30303-3083, USA
}

\section{CONCEPT}

Because of the improvements in CCD detector quality and availability, as well as the increased performance and lower prices of PC compatible computers and digitizer boards, the time is ripe for getting amateur astronomers interested in speckle interferometry. Presented is a low-cost (under $\$ 1500$ U.S., without computer) prototype astrometric speckle interferometry package suitable for use by amateur astronomers on their own telescopes. Table 1 lists the names and addresses of the manufacturers of the various products required as well as their prices.

TABLE 1. Manufacturers and cost estimate.

Edmund Scientific Company

101 E. Gloucester Pike, Barrington, NJ 08007-1380

Telephone: (609)573-6259

4X Microscope Objective, Stk. No. A38,345

535-700nm Filter, Stk. No. A43,386

Amperex Electronic Company

A Division of North American Philips Corporation

Imaging Products Business Unit

Providence Pike, Slatersville, RI 02876

Telephone: (401)762-3800

CCD Monochrome Imaging Module, type 56471

$\$ 440.00$

Catenary Systems

470 Belleview, St. Louis, MO 63119

Telephone: (314)962-7833

Image Processing Library and Digitizer Card

$\$ 490.00$

IDEC, Inc.

1195 Doylestown Pike, Quakertown, PA 18951

Telephone: (215)538-2600

Manufacturer of Digitizer Card available through Catenary Systems

Total Price for Components

$\$ 978.70$ 


\section{OBSERVATIONS AND REDUCTIONS}

The speckle package was tested using a $0.2-\mathrm{m}$ (8-inch) $\mathrm{f} / 10 \mathrm{Schmidt-Cassegrain}$ telescope on 24 Mar, 1992 at GSU's Hard Labor Creek Observatory (HLCO). The binary systems of Castor ( $\alpha \mathrm{Gem})$ and Algieba ( $\gamma$ Leo) were observed, both with a 16X microscope ob jective and no filter. The package was also tested using the 0.4-m (16-inch) f/16 classical Cassegrain at HLCO on $31 \mathrm{Mar}, 1992$. The binary systems of Algieba ( $\gamma$ Leo), Castor ( $\alpha \mathrm{Gem})$, Mizar ( $\zeta \mathrm{UMa})$, and $\pi$ Boo, were all observed without a microscope objective and with a red $(610-690 \mathrm{~nm})$ filter. Additionally, Algieba was observed with a green $(530-570 \mathrm{~nm})$ filter. The data from both instruments were recorded on $8 \mathrm{~mm}$ video cassette.

The directed vector autocorrelation (DVA) was done using the CHARA DVA setup (an Imaging Technology PC Vision+ digitizer card in a 386 PC to digitize and store the bright pixel positions and UNIX workstations to perform the DVA, see Bagnuolo et al. 1992). To approximate the capability of an anticipated amateur DVA setup (a simple digitizer card in a $386 \mathrm{PC}$, calculating the DVA real-time) and a 10 minute integration, only the brightest 100 pixels in each of about 1800 frames were used in the DVA.

The secondary positions of the binaries were determined using the CHARA astrometric reduction routines written in Interactive Display Language (IDL). Peaks were found in all DVA's except that of $\pi$ Boo. The results are summarized in Table 2.

TABLE 2. Selected binaries.

\begin{tabular}{|cccccccc|}
\hline Name & $\mathrm{V}$ & $\Delta \mathrm{m}$ & $\begin{array}{c}\text { Aperture } \\
(\mathrm{m})\end{array}$ & $\begin{array}{c}\text { Image Scale } \\
\text { (pix/arcsec) }\end{array}$ & $\begin{array}{c}\text { Filter } \\
(\mathrm{nm})\end{array}$ & $\begin{array}{r}\text { Fitted X } \\
\text { (pixels) }\end{array}$ & $\begin{array}{r}\text { Fitted Y } \\
\text { (pixels) }\end{array}$ \\
\hline Algieba & 1.90 & 1.25 & 0.4 & 1.936 & 650 & -6.85 & -5.69 \\
Algieba & 1.90 & 1.25 & 0.4 & 1.936 & 550 & -6.54 & -5.38 \\
Algieba & 1.90 & 1.25 & 0.2 & 5.229 & - & 5.73 & 22.82 \\
Castor & 1.58 & 0.86 & 0.4 & 1.936 & 650 & 4.32 & -1.75 \\
Castor & 1.58 & $\mathbf{0 . 8 6}$ & 0.2 & 5.229 & - & 0.16 & -6.17 \\
Mizar & 2.06 & 1.68 & 0.4 & 1.936 & 650 & -10.97 & -25.82 \\
$\pi$ Boo & 4.54 & 0.92 & 0.4 & 1.936 & 650 & - & - \\
\hline
\end{tabular}

\section{CONCLUSION}

In speckle interferometry, the brightness of the speckle is independent of the telescope size. However, the speckle size is inversely proportional to the telescope size, scaling as the Airy disk. Increasing the effective focal ratio of a telescope through the use of microscope objectives has the effect of enlarging the seeing disk as well as decreasing the available light per pixel in the CCD camera. Therefore, finding the optimum image scale for calculating the astrometry while still getting enough light per pixel is the greatest challenge. In practice, the Airy disk should certainly be no more than 10 pixels across. 
Three of the more popular sizes of amateur telescopes are the 8-in (20.32$\mathrm{cm})$, the 13.1-in $(33.33-\mathrm{cm})$, and the 17.5 -in $(44.45-\mathrm{cm})$. Table 3 lists the diffraction limits in arc-seconds, the number of speckles across the seeing disk for average seeing, the magnitude limits with filters in place, and an approximate number of known binaries that could be measured for each of these telescopes. The magnitude limits for two different image scales are given to highlight the cost of higher magnification.

TABLE 3. Telescope comparisons.

\begin{tabular}{|cccccc|}
\hline $\begin{array}{c}\text { Aperture } \\
(\mathrm{cm})\end{array}$ & $\theta_{d}$ & $\begin{array}{c}\text { No. of } r_{0} \\
\left(r_{0}=10 \mathrm{~cm}\right)\end{array}$ & $\begin{array}{c}\text { V limit at } \\
\text { 2 pix/arcsec }\end{array}$ & $\begin{array}{c}\mathrm{V} \text { limit at } \\
\text { 4 pix/arcsec }\end{array}$ & $\begin{array}{c}\text { No. of Objects } \\
\text { at 2 pix/arcsec }\end{array}$ \\
\hline 20.3 & $0^{\prime \prime} .77$ & 4 & 3.7 & 2.2 & 9 \\
33.3 & $0^{\prime \prime} .47$ & 11 & 4.8 & 3.3 & 29 \\
44.5 & $0^{\prime \prime} .38$ & 19 & 5.4 & 3.9 & 53 \\
\hline
\end{tabular}

\section{REFERENCES}

Bagnuolo, W.G. Jr., Mason, B.D., Barry, D.J., Hartkopf, W.I., \& McAlister, H.A. 1992, AJ, 103, 1399 